A LETTERS JOURNAL EXPLORING THE FRONTIERS OF PHYSICS

\title{
OFFPRINT
}

\section{Feedback characteristics of nonlinear dynamical systems}

A. Lahellec, S. Hallegatte, J.-Y. Grandpeix, P. Dumas and S. BLANCO

EPL, 81 (2008) 60001

Please visit the new website

www.epljournal.org 


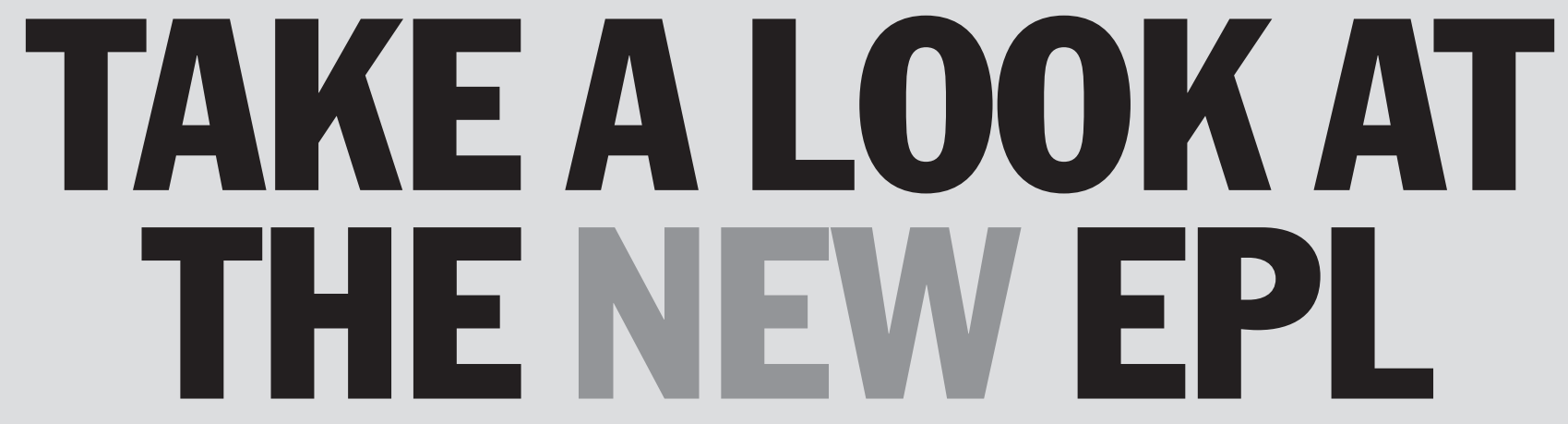

\section{Europhysics Letters (EPL) has a new online home at www.epljournal.org}

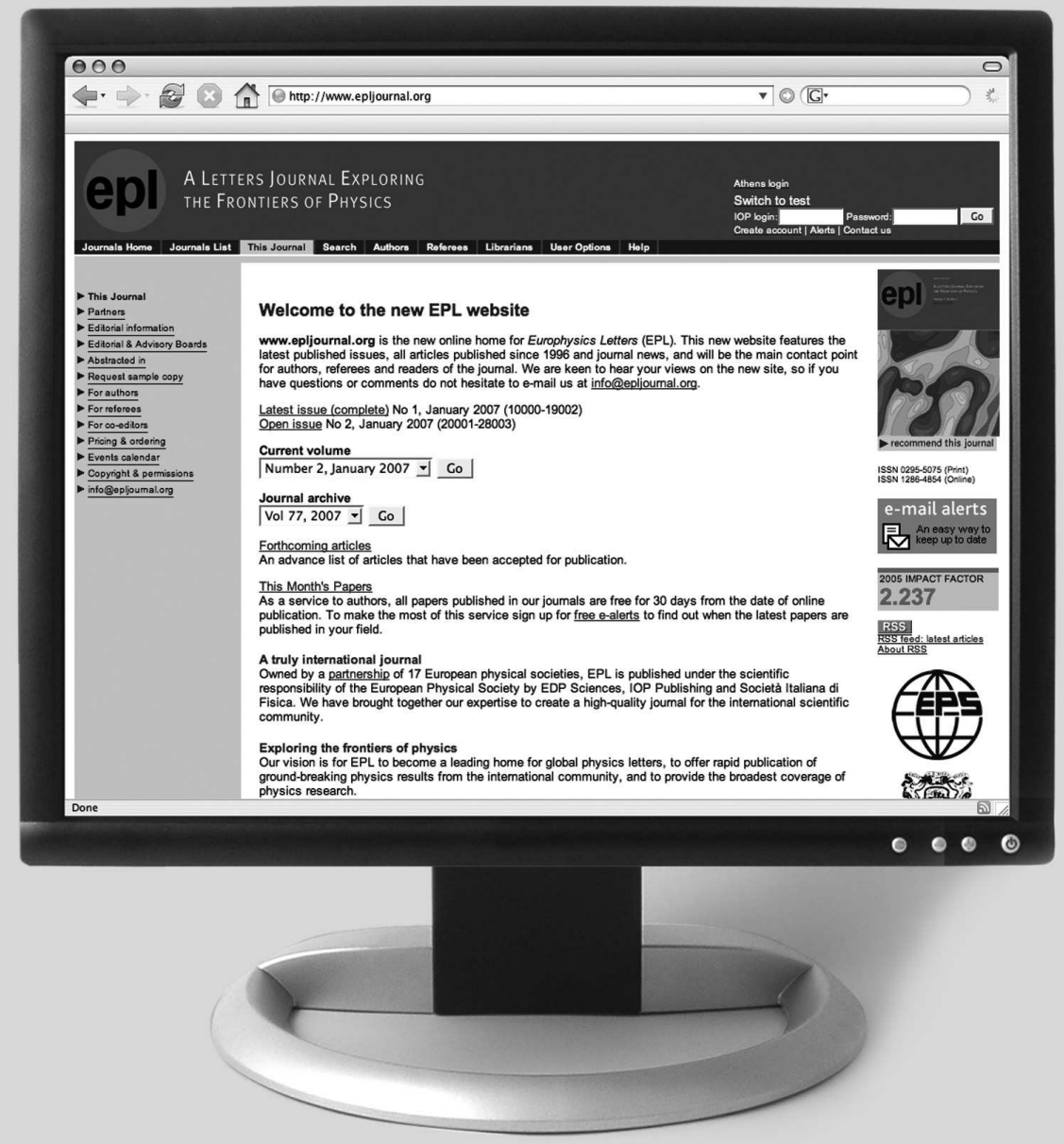

Take a look for the latest journal news and information on:

- reading the latest articles, free!

- receiving free e-mail alerts

- submitting your work to EPL 


\title{
Feedback characteristics of nonlinear dynamical systems
}

\author{
A. Lahellec ${ }^{1(a)}$, S. Hallegatte ${ }^{2,3}$, J.-Y. Grandpeix ${ }^{1}$, P. Dumas ${ }^{4}$ and S. Blanco ${ }^{5}$ \\ ${ }^{1}$ Laboratoire de Météorologie Dynamique, CNRS, Université Paris-VI - Paris, France \\ ${ }^{2}$ Centre International de Recherche sur l'Environnement et le Développement, ENPC - Paris, France \\ ${ }^{3}$ Ecole Nationale de la Météorologie, Météo-France - Toulouse, France \\ ${ }^{4}$ Environment Research and Teaching Institute, Ecole Normale Supérieure - Paris, France \\ ${ }^{5}$ Laboratoire Plasma et Conversion d'Energie (LAPLACE, CNRS-UPS), Université de Toulouse - Toulouse, France
}

received 29 August 2007; accepted in final form 18 January 2008

published online 20 February 2008

PACS 05.45.-a - Nonlinear dynamics and chaos

PACS 92.70.Gt - Climate dynamics

\begin{abstract}
We propose a method to extend the concept of feedback gain to nonlinear models. The method is designed to dynamically characterise a feedback mechanism along the system natural trajectory. The numerical efficiency of the method is proved using the Lorenz (1963) classical model. Finally, a simple climate model of water vapour feedback shows how nonlinearity impacts feedback intensity along the seasonal cycle.
\end{abstract}

Copyright (C) EPLA, 2008

Introduction. - Since its introduction by Bode [1], the concept of feedback gain has proved a very efficient tool in the engineering fields. Originally elaborated in the frequency domain for linear systems, it further allowed for the boom in techniques of optimal control that have indeed been extended to nonlinear systems in the $60 \mathrm{~s}$ - robust filtering, optimal or adaptive control, etc. (e.g. [2], and the references therein). It also inspired a variety of research applications for controlling or synchronising chaotic systems using feedback laws (see [3]).

Our concern here is different: the concept of feedback is thought as a way to gain insight into the behaviour of a system. In this vein, feedback is frequently mentioned in biology, in chemistry, in physics, and in economics (e.g. the Keynes feedback, in [4]), but usually on a qualitative basis only: one will essentially speak of positive (destabilising) or negative (stabilising) effect of a feedback mechanism. Manabe and Wetherald [5] originated a breakthrough in climate sciences when they proposed an analysis of the Earth climate allowing to assess in terms of quantitative feedback gain the relative importance of various contributing phenomena. From then on, the study of global warming, caused by the increasing concentration of greenhouse gases like $\mathrm{CO}_{2}$, has been systematically analysed in terms of feedback processes (water vapour, clouds, albedo ...), as in [6-9] among many others. Nevertheless, there is still ambiguity about a general definition of feedback gain, as analysed in [10], where four definitions are found.

\footnotetext{
(a) E-mail: alain@lmd.jussieu.fr
}

Considering the role of water vapour feedback (WVF) in global warming, the direct climate response to an increase in $\mathrm{CO}_{2}$ concentration is an increase in the mean atmospheric temperature $\left(T_{\mathrm{atm}}\right)$, due to a change in the long-wave radiation budget. Following this direct effect, the feedback process runs as follows: since climatic mechanisms maintain relative humidity unchanged, the atmospheric warming translates into a larger water vapour content in the atmosphere. Being water vapour a strong greenhouse gas, the initial $T_{\text {atm }}$ perturbation is amplified. From the comparison between the equilibrium response obtained in climate models when including that feedback and the similar response when artificially suppressing that feedback, it is commonly said that "WVF increases by 60 percent the warming due to carbon dioxide perturbation".

A major limitation of this approach is that it ignores the dynamical aspects of Bode's original concept since only the difference between two systems at equilibrium is considered. Indeed, for systems that take very long to reach a new equilibrium - as the climate systemthe dynamics are of primary importance. In [11], the authors explained how to retrieve the dynamics of feedback for systems between two equilibria. Regarding the $\mathrm{CO}_{2}$ increase, they showed with an idealised model that the atmospheric WVF response is negative on the short term (damping effect), and only becomes positive (amplifying effect) after a build-up period of four to seven years, where the 60 percent increase is finally reached at the perturbed equilibrium. 
This equilibrium approach is nevertheless a severe limitation. Indeed, climate is driven by natural forcing (e.g. solar forcing) and can hardly be considered as in equilibrium. The purpose of this article is to extend the feedback concept to dynamical nonlinear systems.

We shall first introduce a generic method to define a feedback loop in a state-space model. Then, the system response to the external perturbation is characterised by a set of dynamical functions related to the feedback gain. We observe that when the system simplifies into autonomous conditions, these functions are the same as those introduced by Bode. A first application on the Lorenz (1963) model is carried out to demonstrate the numerical efficiency of the method on a strongly nonlinear model. Finally, we come back to the water vapour feedback and, using a very simple nonlinear model, we compare the equilibrium response with more realistic trajectories.

Formal background. Consider a state-space model driven by some external forcing $\boldsymbol{h}(t)$ :

$$
\partial_{t} \boldsymbol{\eta}(t)=\boldsymbol{g}(\boldsymbol{\eta}(t), \boldsymbol{h}(t)), \quad \boldsymbol{\eta}(t=0)=\boldsymbol{\eta}_{0},
$$

where $\boldsymbol{\eta}$ is the state vector. Once a reference trajectory $\left(T^{\mathrm{ref}}\right)$ is followed, perturbations to system (1) are solutions of the so-called tangent linear system (TLS):

$$
\partial_{t} \delta \boldsymbol{\eta}(t)=A(t) \delta \boldsymbol{\eta}(t)+B(t) \delta \boldsymbol{h}(t), \quad t \in[0, T] .
$$

In this equation, $\delta \boldsymbol{\eta}$ is the deviation of the perturbed system from the reference trajectory, caused by a perturbation $\delta \boldsymbol{h}(t)$ of the reference forcing function, and $A(t)=$ $\partial_{\eta} \boldsymbol{g}(t)$ and $B(t)=\partial_{h} \boldsymbol{g}(t)$ are the Jacobian matrices. We call circulating tangent linear system (CTLS) the nonautonomous TLS (2), meaning that it is defined along $T^{\text {ref }}$. The classical solution of $(2)$ reads

$$
\left\{\begin{array}{l}
\delta \boldsymbol{\eta}(t)=\Phi(t, 0) \delta \boldsymbol{\eta}(0)+\int_{0}^{t} \Phi(t, \tau) B(\tau) \delta \boldsymbol{h}(\tau) \mathrm{d} \tau \\
\partial_{t} \Phi(t, \tau)=A(t) \Phi(t, \tau), \quad \Phi(\tau, \tau)=I
\end{array}\right.
$$

where $\Phi(t, 0)$ is the state transition matrix of the perturbed system and $\delta \boldsymbol{\eta}(0)$ a perturbation of the initial conditions [12]. Such a system does not exhibit any explicit structure in which a feedback loop can be specified. We now introduce a way to provide a state model with a feedback structure.

\section{Feedback concepts. -}

Feedback loop. A feedback loop is a circular structure that links some chosen physical quantity to the rest of the system. A general method to provide a model with such a structure can be stated as follows:

1) choose an "output" variable from the model that will be used as a test variable - in the WVF case in this letter, we choose $T_{s}$, the atmospheric surface temperature, as the test variable;

2 ) add a perturbed version of this variable to the system - say $\varphi=T_{s}+$ perturbation;

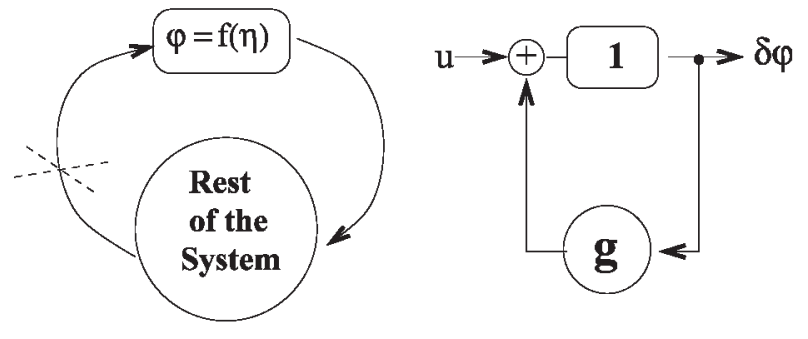

Fig. 1: Feedback structure using an extra-variable $\varphi$ (left panel) and the classical block-diagram (right panel).

3) close the feedback loop: the new variable $\varphi$ is introduced back in the model where the mechanisms are depending on its perturbed version -in eq. (17), the water content is chosen as depending on $\varphi$ instead of $T_{s}$.

Accordingly, the introduction of a feedback loop in the original system corresponds to the following formal procedure:

- a scalar variable $\varphi$ is added to the system with equation $\varphi=f(\eta)$ - see eq. (14) for instance;

- the primitive model (1) is modified to be sensitive to $\varphi$ in such a way that original and modified models are mathematically equivalent:

$$
\partial_{t} \boldsymbol{\eta}(t)=\boldsymbol{g}^{\prime}(\boldsymbol{\eta}(t), \varphi(t), \boldsymbol{h}(t))=\boldsymbol{g}(\boldsymbol{\eta}(t), \boldsymbol{h}(t))
$$

Now, if a scalar perturbation $u(t)$ is applied to the $\varphi$ equation only $(\varphi(t)=f(\eta)+u(t))$, the system will respond to that change within a feedback loop: perturbation $u$ on $\varphi \rightarrow$ selected mechanisms $\rightarrow$ rest of the system $\rightarrow$ response $\delta \varphi$ (see fig. 1).

The opening of the loop means preventing the system from responding on $\varphi$ (cross in fig. 1): $\delta \varphi^{0}=u$. The classical response would be $\delta \varphi=\frac{1}{1-g} u$ in a symbolic form.

The difference between the perturbation $u(t)$ and the response in $\varphi(t)$ is commonly understood as the feedback effect of the system on $\varphi$.

A general definition of dynamical feedback. Following the preceding procedure, the original tangent system (2) is modified into:

$$
\left\{\begin{array}{l}
\partial_{t} \delta \boldsymbol{\eta}(t)=A^{b}(t) \delta \boldsymbol{\eta}(t)+|b(t)\rangle \delta \varphi(t) \\
\delta \varphi(t)=\langle c(t)| \delta \boldsymbol{\eta}(t)+u(t)
\end{array}\right.
$$

The column and row matrices $|b\rangle=\partial_{\varphi} \boldsymbol{g}^{\prime}$ and $\langle c|=\partial_{\eta} f$ formalise the structure of the feedback loop. For system (4) to be mathematically equivalent to (2), the following equality $A(t)=A^{b}(t)+|b\rangle\langle c|(t)$ must hold. Opening the loop corresponds to the cancelling of $\langle c|$, that disallows the system perturbation to influence $\varphi$.

It is easily checked that system (4) takes the form of (2) when $\delta \varphi$ has been eliminated. Correspondingly, we define 
the state sensitivity $\boldsymbol{s}(t, 0)$ as the state response in (4) using solution $(3)-$ setting $\delta \boldsymbol{\eta}(0)=0$ :

$$
\boldsymbol{s}(t, 0)=\delta \boldsymbol{\eta}(t)=\int_{0}^{t} \Phi(t, \tau)|b(\tau)\rangle u(\tau) \mathrm{d} \tau .
$$

We call $\sigma^{u}(t, 0)=\delta \varphi(t)$ the response in $\varphi$, and $\varrho^{u}(t, 0)=$ $\sigma^{u}(t, 0)-u(t)$ the feedback effect of the system on $\varphi$, which is the deviation of the response from the perturbing function starting at $\tau=0$ :

$$
\varrho^{u}(t, 0)=\left\langle c(t)\left|\int_{0}^{t} \Phi(t, \tau)\right| b(\tau)\right\rangle u(\tau) \mathrm{d} \tau .
$$

This function can be computed along $T^{\text {ref }}$ from the system obtained by time-differentiating eq. (5):

$$
\left\{\begin{array}{l}
\partial_{t} \boldsymbol{s}(t, 0)=A^{b} \boldsymbol{s}(t, 0)+|b\rangle \varrho^{u}(t, 0)+|b\rangle u(t), \\
\varrho^{u}(t, 0)=\langle c| \boldsymbol{s}(t, 0), \quad \boldsymbol{s}(0,0)=\mathbf{0} .
\end{array}\right.
$$

In [13], Cacuci has shown how to obtain such systems using the Gâteaux differentiation, and called "general sensitivity" the response $\delta \varphi$ to $u$.

It is worth noticing that the CTLS integration of (7) is carried out simultaneously with the reference trajectory, that is, without applying any actual numerical perturbation that would change the trajectory. This is admissible as far as the perturbation amplitude stays within the limits of linearity of the perturbed system. Also, the CTLS provides a definite advantage concerning the generic features of the results, compared to finite difference methods using finite perturbation amplitudes.

The autonomous limit. On short segments of trajectory, the constant Jacobian matrices define a local TLS, with a simpler state transition matrix: $\Phi^{s}(t, \tau)=$ $\exp A(t-\tau)$. For such autonomous system, a Laplace transformation can be applied to the convolution product in (6) to obtain:

$$
\tilde{\varrho}_{s}^{u}(\mu)=\left\langle c\left|(\mu I-A)^{-1}\right| b\right\rangle \tilde{u}(\mu),
$$

where $\tilde{f}(\mu)$ is the Laplace transform of $f(t)$, with $\mu$ as the Laplace variable. We call standing this function corresponding to the dynamical response of the local autonomous system to perturbation. When the system reaches equilibrium, the CTLS becomes truly autonomous, and the standing response identifies with the circulating one, a property that allowed the dynamical analysis in [11] simply using the inversion of eq. (8).

To retrieve the feedback gain, one can directly apply a Laplace transformation to the autonomous version of eq. (4) to get

$$
\left\{\begin{array}{l}
\left(\mu I-A^{b}\right) \tilde{\boldsymbol{s}}(\mu)=|b\rangle \tilde{\sigma}(\mu) \\
\tilde{\sigma}(\mu)=\langle c| \tilde{\boldsymbol{s}}(\mu)+\tilde{u}(\mu)
\end{array}\right.
$$

which, after elimination of $\tilde{\boldsymbol{s}}$, leads to

$$
\left(1-\left\langle c\left|\left(\mu I-A^{b}\right)^{-1}\right| b\right\rangle\right) \tilde{\sigma}(\mu)=\tilde{u}(\mu)
$$

The form $(1-\tilde{g}(\mu)) \tilde{\sigma}(\mu)=\tilde{u}(\mu)$ of eq. (10) is the one used by Bode as the definition of feedback gain in the Laplace domain:

$$
\tilde{g}(\mu)=\left\langle c\left|\left(\mu I-A^{b}\right)^{-1}\right| b\right\rangle
$$

In this form, it is easily seen that setting $\langle c|=0$ corresponds to the open-loop mode, that is, to a null feedback gain.

We may conclude that the two feedback functions $\sigma$ and $\varrho$ generalise the autonomous ones with the following correspondence:

$$
\left\{\begin{array}{l}
\sigma^{u}(t, 0) \longleftrightarrow \tilde{\sigma}(\mu)=\frac{1}{1-\tilde{g}(\mu)} \tilde{u}(\mu), \\
\varrho^{u}(t, 0) \longleftrightarrow \tilde{\varrho}(\mu)=\tilde{u}(\mu)-\tilde{\sigma}(\mu)=\frac{\tilde{g}(\mu)}{1-\tilde{g}(\mu)} \tilde{u}(\mu) .
\end{array}\right.
$$

Dynamical feedback characteristics. The standard definition of feedback characteristic functions $\sigma^{\Upsilon}$ and $\varrho^{\Upsilon}$ considers the particular perturbing step-function $u(\tau)=$ $\Upsilon(\tau)$, whose Laplace transform is $\tilde{\Upsilon}(\mu)=\frac{1}{\mu}$ in (12). Correspondingly, the feedback effect characteristic function $\varrho^{\Upsilon}$ is given by eq. (6) setting $u(\tau)=1$. This form is referred to as the "F4 sensitivity-altering" feedback in [10].

Unlike the linear feedback mechanism which is completely characterised by autonomous functions, the nonlinear case is depending both on a trajectory and on a starting point. It may happen that, in practical applications, those primitives are known: in an economic model, a shock (i.e. on oil prices) arises at a known date; in climate models, a volcano eruption is also chronologised. In general the feedback response may strongly depend on the initial time of perturbation: this pertains to the richness of nonlinear systems behaviour. A comparison between standing (8) and circulating (6) TLS functions is presented in the application section.

As a final formal remark, the Dirac impulse $u(\tau)=\delta(\tau)$ may also be useful as a perturbing function because the corresponding $\varrho^{\delta}(t, \tau)$ kernel can be convoluted with any perturbing function $v(t)$ to produce the model response: $\delta \varphi(t)=\langle c(t)| \int_{0}^{t} \varrho^{\delta}(t, \tau) v(\tau) \mathrm{d} \tau+v(t)$. Because in that case $\varrho^{\delta}(t, \tau)$ is needed as a function of $\tau$, the feedback system (7) shall be integrated in its adjoint-system form, see [14]. That feedback form corresponds to the "F3 stability-altering" class in [10].

Circulating feedback gain function. Contrary to the linear case, one cannot retrieve the feedback gain of nonlinear systems from neither $\varrho$ nor $\sigma$, as in (12). A particular system has to be built. Let us consider the following system:

$$
\left\{\begin{array}{l}
\partial_{t} s(t, 0)=A^{b} s(t, 0)+|b\rangle \Upsilon(t) \\
g(t, 0)=\langle c| s(t, 0)
\end{array}\right.
$$

In the autonomous case, that system simplifies into: $\tilde{g}(\mu)=$ $\mu^{-1}\left\langle c\left|\left(\mu I-A^{b}\right)^{-1}\right| b\right\rangle$, that is recognised as Bode's feedback gain. System (13) is the so-called "open-loop" mode which provides for the general feedback gain function in its circulating form. 


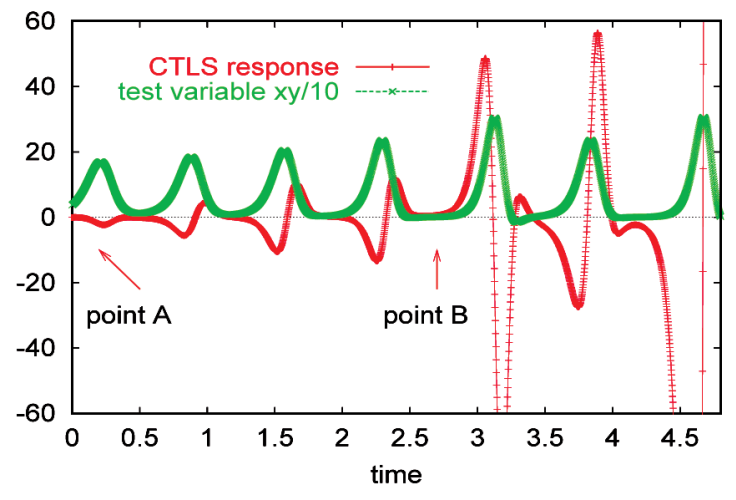

Fig. 2: Test variable in the Lorenz model and feedback effect CTLS function.

Application to the Lorenz model. - We illustrate the method using the Lorenz [15] model to prove the numerical efficiency of the method on a well-known nonlinear system. The system - modified into feedback form- reads

$$
\left\{\begin{array}{l}
\partial_{t} x=s(y-x), \\
\partial_{t} y=(r x-y-x z), \\
\partial_{t} z=-b z+\varphi \\
\varphi=x y
\end{array}\right.
$$

with the set of parameters $s=10, r=28, b=\frac{8}{3}$ that leads to a chaotic behaviour (fig. 5 displays the $x, y$ trajectory with the two wings of the attractor).

To build the feedback form (14), we have chosen $\varphi=x y$ as a nonlinear test variable, but any other term could have been chosen, since we are not interested in physical analysis here ${ }^{1}$. Also, the comments on dynamics will only consider numerical aspects of the results.

Figure 2 shows the evolution of $\varphi$ and the CTLS $\varrho^{\Upsilon}(t, 0)$ function along the trajectory, starting at the beginning of an orbital point $A$ and going after four revolutions through a transition point B (in the following, $\Upsilon$ is dropped from symbol $\left.\varrho^{\Upsilon}\right)$.

Because of the symmetry of the system across $z$, the test variable $\varphi=x y$ does not clearly reveal the transition, whereas the CTLS feedback effect does. Between points A and $\mathrm{B}$ in the figure, the interaction mode responds with a zigzag at each orbit, increasing regularly in amplitude. After B the response at transition amplifies.

This systematic amplification before transitions is seen in fig. 3 which displays the long-term tendency of $\varrho(t, 0)$. The chaotic nature of the model is correctly characterised by the average linear increase of the log CTLS effect which is found to follow a local Lyapunov exponent. Moreover, the oscillation is similarly increasing in amplitude, an aspect that is not revealed by the Lyapunov analysis.

Figure 4 illustrates what happens when starting far from the attractor. Clearly, the feedback effect is very

\footnotetext{
${ }^{1}$ Nevertheless, a physical interpretation can be found in http://www.lmd.jussieu.fr/ZOOM/doc/examples.html.
}

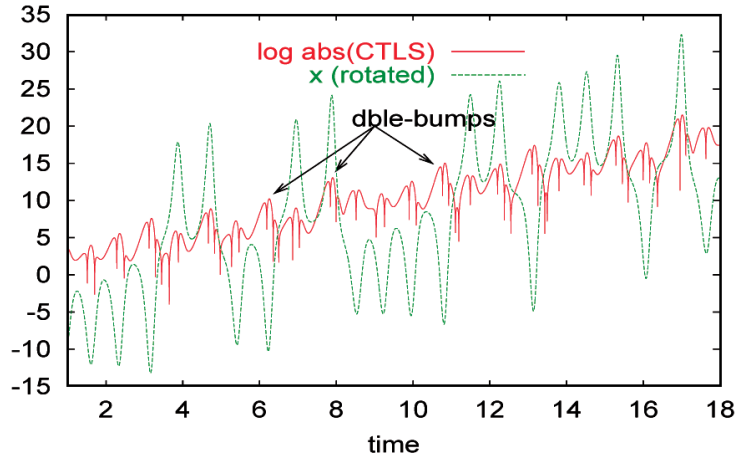

Fig. 3: Long-term feedback effect (log scale). The $x$ trajectory is rotated along the Lyapunov exponent: $x \rightarrow x+\lambda_{L} t$, with $\lambda_{L} \simeq 0.95$, to ease curve collocation.

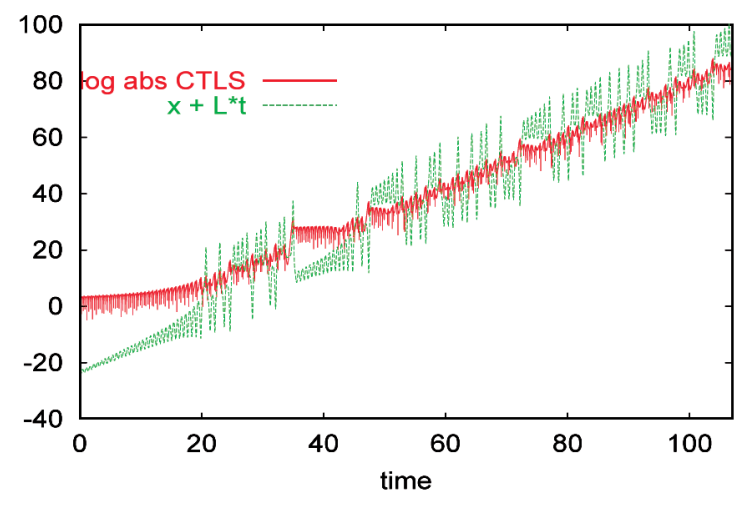

Fig. 4: Same as fig. 3 with initial conditions out of the attractor.

different depending on whether the current point is lying within the attractor or not: the average increase of $\varrho(t, 0)$ reduces each time the circulating point leaves the attractor (see times $0,35,48,73$ ). The attractor can therefore be characterised by an enhanced action of the $x y$ mode interaction.

Short-term dynamical response. The asymptotic behaviour of the feedback function shows coherence with the Lyapunov stability analysis, but feedback analyses are further concerned with short- and medium-term dynamics. To account for the Lyapunov divergence of the Lorenz system, we will remove this divergence by applying a factor $\exp \left(-\lambda_{L} t\right)$ on dynamical functions.

Let now zoom on the four-orbits sequence going to transition. In fig. 5 , the $x, y$ trajectory is plot with error bars. Error bars represent the CTLS effect amplitude (log-scaled), plotted as blue vertical when above the mean Lyapunov increase, and as green horizontal when under the mean Lyapunov increase. Both orbits are followed clockwise. The first (inner) orbit shows the under effect only, when the point is orbiting around the left-wing point of steady convection (at the centre of orbits).

Between points 1 and 2 on the fourth orbit, the above reaction is active when the movement decelerates toward the perigee (close to central point $(0,0)$ ), and when it 


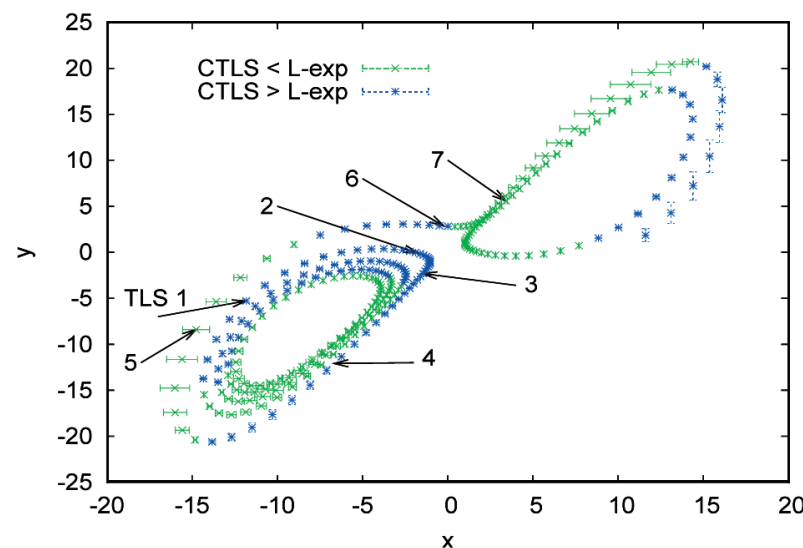

Fig. 5: Lorenz attractor in the $(x, y)$-plane; error bars: CTLS effect (scaled, see text).

accelerates heading to the apogee (see points 3 and 4). This suggests that the above effect is determined by a repulsion from the central point. The transition initiates with a strong under reaction after the apogee (5), and the circulating-point decelerates again from the central-point repulsive effect, but with an offset that knocks the state onto the right wing.

Such dynamical characteristics show how different regions of the attractor influence the behaviour of the system. The feedback formalism just brings another approach, simple and general, to the numerous and sophisticated tools developed for nonlinear analysis.

Water vapour feedback model. - Coming back to the water vapour feedback effect, we have built the simplest model to analyse the slow response of climate to a $\mathrm{CO}_{2}$ perturbation that matches the results obtained in [11]. The purpose of the exercise is to give an idea of what importance nonlinear mechanisms might have on WV feedback.

We consider the evolution of a $30 \mathrm{~m}$ thick mixed ocean layer, irradiated by a mean solar flux $F_{S W}=343 \mathrm{Wm}^{-2}$. The energy loss is from the LW radiative flux only:

$$
C_{o} \frac{\mathrm{d} T_{s}}{\mathrm{~d} t}=F_{S W}-F_{L W}
$$

with $C_{o}=1.257 \cdot 10^{8} \mathrm{JK}^{-1} \mathrm{~m}^{-2}$.

To simulate the effect of greenhouse gases (WV or $\mathrm{CO}_{2}$ ) on radiation, we use an equivalent radiation altitude $z_{e}$. Seen from space, this altitude gives an equivalent outcoming blackbody radiation temperature due to both surface and low-level layers of the atmosphere (that contain a substantial amount of water vapour):

$$
F_{L W}=\sigma\left(T_{s}-z_{e} \gamma_{z}\right)^{4}
$$

where $\sigma=5.67 \cdot 10^{-8} \mathrm{Wm}^{-2} \mathrm{~K}^{-4}$ is the StephanBoltzmann's constant and $\gamma_{z}=6.5 \mathrm{Kkm}^{-1}$ the fixed

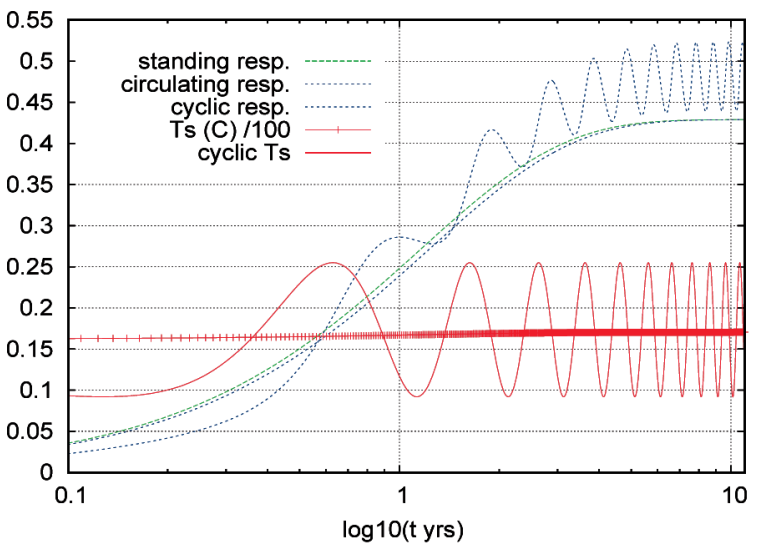

Fig. 6: Water vapour feedback model responses.

atmospheric-temperature lapse rate (i.e. $\left.\gamma_{z}=\partial_{z} T_{\mathrm{atm}}\right)$. The specific humidity at surface $Q$ is given by the Clausius-Clapeyron relation:

$$
Q=f\left(T_{s}\right)=\mu \exp \left[\frac{L_{v}}{R_{m}}\left(\frac{1}{T_{0}}-\frac{1}{T_{s}}\right)\right]
$$

with $\mu=11.05, \frac{L_{v}}{R_{m}}=5423 \mathrm{~K}$ and $T_{0}=273.15 \mathrm{~K}$.

We now determine the dependence of $z_{e}$ on $Q$. When the ocean temperature $T_{s}$ increases due to a $\mathrm{CO}_{2}$ concentration increase, more water evaporates in the atmosphere, and $z_{e}$ increases: $z_{e}=z_{0}+\alpha Q$. Parameters $z_{0}=1 \mathrm{~km}$ and $\alpha=0.02$ have been calibrated from the more complete model in [11], as they account for the elevation of $z_{e}$ due to the amount of water vapour in low atmosphere, which is non-trivially related to $Q$.

To build the WV feedback loop, we define the new variable as $\varphi=T_{s}$, and let the atmospheric water vapour depend on it in eq. (17): $Q=f(\varphi)$. In this new structure of the system, $\varphi$ is the air-surface temperature, and can now be perturbed independently of $T_{s}$.

The WV feedback loop is: $\varphi \uparrow \rightarrow Q \uparrow \rightarrow z_{e} \uparrow \rightarrow L W \downarrow \rightarrow$ $T_{s} \uparrow \rightarrow \varphi$ and it increases more (positive feedback). The open-loop mode is obtained when $Q$ does not respond to the change in $\varphi$.

We first consider a $T_{s}$ trajectory starting out of equilibrium from $16^{\circ} \mathrm{C}$, and slowly reaching equilibrium at $17^{\circ} \mathrm{C}$ (red-points curve in fig. 6). At the final equilibrium, the standing response to a perturbation in $\varphi$ is given by the blue curve, an exponential response that reaches 0.43 with an $e$-folding time of 423 days. The corresponding feedback gain is $30 \%$ as in [11] (the model is calibrated for that purpose). This means that if a step in $\mathrm{CO}_{2}$ concentration is responsible for a final increase of $1 \mathrm{~K}$ in the model temperature, then an immediate fraction of $0.7 \mathrm{~K}$ is due to the direct $\mathrm{CO}_{2}$ effect, while the $\mathrm{WV}$ feedback is gradually adding an extra $0.3 \mathrm{~K}$ (i.e. $0.7 \times 0.43)$.

The response time is nevertheless much shorter than the several years in [11], this being due to the absence of 
delay in the coupling of the ocean to the atmosphere in this crude model, where the response time is proportional to $C_{o}$ - or to the ocean layer thickness ${ }^{2}$.

The CTLS response is the green curve just above with the same asymptotic response of 0.43 . Following the trajectory, the circulating response is very close to the standing one, meaning that the nonlinearity of the model is marginally active.

We now consider a cyclic seasonal SW forcing with an added amplitude of $\pm 100 \mathrm{Wm}^{-2}$; the response is the blue oscillating curve and the thin red line is the surface temperature trajectory, finally oscillating between $9{ }^{\circ} \mathrm{C}$ and $25^{\circ} \mathrm{C}$. The nonlinear effect of the model is illustrated by a stronger $\mathrm{WV}$ feedback in the mean. Moreover, it is even stronger during autumn $(\varrho \simeq 0.52)$ than in spring (0.44).

If this minimalist model of climate were realistic, it would tell that in response to a constant $\mathrm{CO}_{2}$ step, fall temperatures would be more amplified than spring temperatures. Such asymmetry proceeds from the nonlinearity of the model.

Final remarks. - We have shown how to extend to nonlinear systems the original Bode's approach of the feedback analysis. Using the tangent linear system along trajectories gives rise to a natural extension of linear concepts and allows to exhibit the dynamical aspects of feedback characteristic functions.

In practical applications, the addition of an extravariable to the basic state model as we did is a simple way to unambiguously build a feedback loop structure in a model, including the explicit reference to the openloop mode. Moreover, this new variable offers flexibility in implementing meaningful feedback loops. In gridded models for instance, a meaningful response relies on the average of an ensemble of grid variables, because the space discretisation is guided by numerical rather than physical concern. A combination of state-space-averaging methods

\footnotetext{
${ }^{2}$ The autonomous response can be analytically obtained from the application of eq. (8) and inversion. One gets the asymptotic feedback gain: $g_{\infty}=\alpha \gamma_{z} \frac{L_{v}}{R_{m}} \frac{Q\left(T_{s}\right)}{T_{s}^{2}}$ and an $e$-folding time of $\tau=$ $\frac{1}{\left(1-g_{\infty}\right) T^{3}\left(z_{e}\right)} \frac{C_{o}}{4 \sigma \gamma_{z}}$ for the response.
}

with feedback characterisation would bring important gain in gridded-systems analysis.

As a conclusion, the proposed CTLS approach offers a technique to apply dynamical feedback concepts to nonlinear models while reflecting some generic features of the results. Moreover, the dynamical aspects of feedback mechanisms address the basic issues in the behaviour of complex systems, especially the effect of interdependence of parts in the functional complexity of the whole system.

$$
* * *
$$

AL wishes to thank J. L. Dufresne, M. Ghil, J. I. YANO and two anonymous referees for useful comments, and especially his daughter JULIE LAHELLEC for the English version.

\section{REFERENCES}

[1] Bode H. W., Network Analysis and Feedback Amplifier Design (Van Nostrand, New York) 1945.

[2] Gupta M. and Sinha N. (Editors), Intelligent Control Systems: Theory and Applications (IEEE Press) 1996.

[3] Отt Edward, Chaos in Dynamical Systems (Cambridge University Press) 2002.

[4] Chiarella C. and Flaschel P., Keynesian Monetary Growth: Macro Foundations (Cambridge University Press) 2000.

[5] Manabe S. and Wetherald R., J. Atmos. Sci, 24 (1967) 241.

[6] Bony S. et al., J. Clim., 19 (2006) 3445.

[7] Cess R. D. et al., Science, 245 (1989) 513.

[8] Hansen J. E. et al., in Climate Processes and Climate Sensitivity, edited by HANSEN J. E. and TAKAhashi T., Vol. 5 (AGU) 1984, p. 130.

[9] Schneider E. K., J. Atmos. Sci., 29 (1972) 1413.

[10] Bates J. R., Q. J. R. Meteorol. Soc., 133 (2007) 545.

[11] Hallegatte S., Lahellec A. and Grandpeix J.-Y., J. Atmos. Sci., 63 (2006) 1878.

[12] Luenberger D. G., Introduction to Dynamic Systems (John Wiley \& Sons) 1979.

[13] Cacuci D. G., J. Math. Phys., 22 (1981) 2794.

[14] Cacuci D. G. and Hall M. C. G., J. Atmos. Sci., 41 (1984) 2063.

[15] Lorenz E. N., J. Atmos. Sci., 20 (1963) 130. 